\section{Systematische Dokumentation zur Ernährung von älteren Patienten gefordert}

\begin{abstract}
Mangel- und Unterernährung bei geriatrischen Patienten treten sogar in den reichen Industriestaaten häufig auf. Als mögliche Ursache dafür werden in internationalen Studien häufig Defizite in der Dokumentation genannt. Eine norwegische Studie hat dies nun genauer untersucht.
\end{abstract}

$\mathrm{O}$ bwohl eine ausreichende Ernährung ein grundlegendes Menschenrecht ist, stellt in Westeuropa die Mangelernährung ein häufiges Problem bei älteren hospitalisierten Patienten dar. Da Studien darauf hinweisen, dass eine Ernährungsdokumentation die Situation verbessern könnte, jedoch noch unzureichend erfolgt, wurde diese in Oslo, Norwegen, detaillierter untersucht. Auch in dieser qualitativen Studie war die Dokumentation der Ernährung bei älteren Patienten sowohl im stationären Bereich als auch beim Übergang vom stationären in den ambulanten Bereich unzureichend. Für die Untersuchung wurde ein hermeneutisch-phänomenologischer Ansatz gewählt: Die Autoren führten Fokusgruppen-Interviews mit 16 Pflegekräften einer großen Universi-

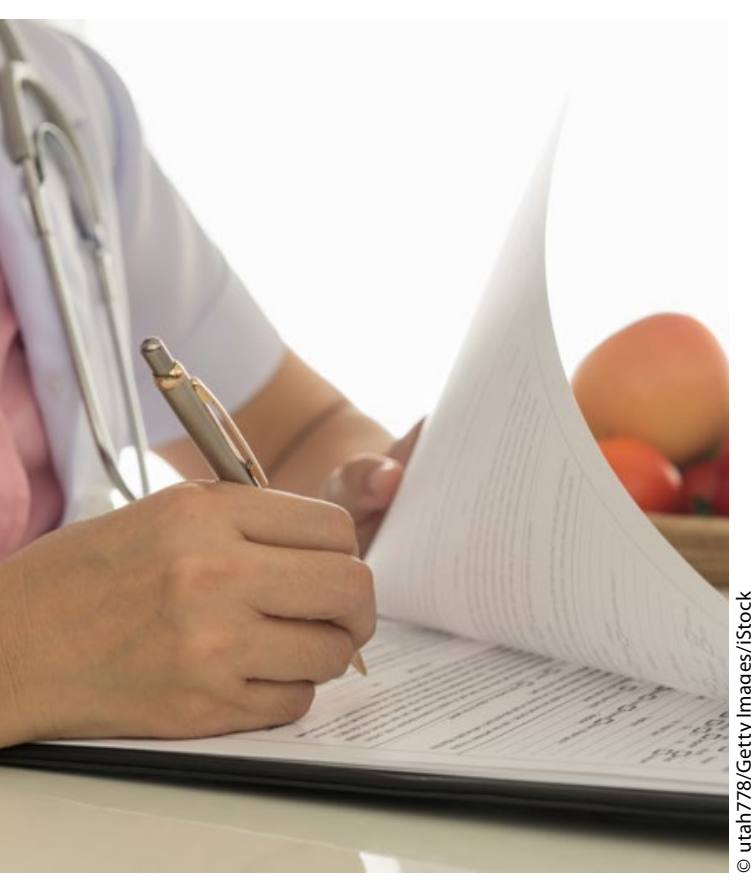

Eine systematische und aufmerksame Ernährungsdokumentation reduziert das Risiko einer Mangelernährung. tätsklinik sowie 27 Pflegekräften in Pflegeheimen, die mit dem Universitätsklinikum kooperieren. Die Teilnehmer der Universitätsklinik stammten aus sieben chirurgischen und internistischen Abteilungen, die alle Patienten der kooperierenden Pflegeheime betreuen. Der Einzugsbereich des Universitätskrankenhauses und der kooperierenden Pflegeeinrichtungen repräsentiert ungefähr $10 \%$ der norwegischen Bevölkerung in städtischen und ländlichen Gebieten. Die Ergebnisse ließen sich drei Hauptthemen zuordnen:

1. Bei der stationären Aufnahme erfolgte keine Dokumentation des ernährungsbedingten Risikos mittels validierter Screening-Tools; die Dokumentation zur Ernährung war unsystematisch und unzureichend.

2. Während des stationären Aufenthalts wurden Informationen zur Ernährung der Patienten nur unzureichend und unsystematisch in der Krankenakte festgehalten; die Aufmerksamkeit gegenüber der Dokumentation dieser Informationen war begrenzt.

3. Zwischen dem Krankenhaus und den Pflegeheimen fand von beiden Seiten nur eine begrenzte Kommunikation zu Informationen über die Ernährung der Patienten statt.

Auf Grund der Ergebnisse halten es die Autoren für wichtig, Systeme zu implementieren, die es den Pflegekräften ermöglichen, ein ernährungsbedingtes $\mathrm{Ri}$ siko zu identifizieren um entsprechende Gegenmaßnahmen ergreifen zu können. Denn eine schlechte Dokumentation kann eine Spirale in Gang setzen, die zu einem erhöhten Risiko für schwere Gesundheitskomplikationen führt.

(pe)

Halvorsen K, et al. Documentation and commulized patients: perspectives of nurses and undergraduate nurses in hospitals and nursing homes. BMC Nurs. 2016;15:70 nication of nutritional care for elderly hospita-

\section{KOMMENTAR}

Mangel- und Unterernährung bei geriatrischen Patienten sind auch in deutschen Kliniken und Pflegeeinrichtungen ein Problem. Immer noch gelten zwischen 20 und $50 \%$ der Senioren in Krankenhäusern und bis zu $60 \%$ in Pflegeheimen als mangelernährt, so die Deutsche Gesellschaft für Gastroenterologie, Verdauungs- und Stoffwechselkrankheiten. Zu Recht haben diese erschreckenden Zahlen den Medizinischen Dienst des Spitzenverbandes Bund der Krankenkassen e.V. zu einer Aktualisierung ihrer Grundsatzstellungnahme „Essen und Trinken im Alter" bewegt. Denn längst gibt es anerkannte Kriterien sowie AssessmentInstrumente zur Erfassung und Beurteilung des Ernährungszustandes. Doch immer noch gehören diese in den wenigsten Einrichtungen zur täglichen Praxis. Kostendruck, Personalmangel, Überlastung der Pflegekräfte führen dazu, dass unzureichend dokumentiert wird. Das rächt sich auf Dauer, wie die Forschungsgruppe Geriatrie der Charité Berlin unlängst herausfand. Erhöhter Pflegeaufwand, verzögerte Rekonvaleszenz, deutlich verlängerte Krankenhausaufenthalte, häufigere stationäre Wiederaufnahme als bei normal Ernährten und dadurch langfristig höhere Kosten sind die Folgen.

Notwendig ist deshalb, bei Patienten, Angehörigen, Pflegepersonal, Ärzten, Klinik- bzw. Heimleitung ein höheres Bewusstsein für die Wichtigkeit einer ausreichenden und dauerhaften Ernährungsdokumentation zu schaffen. Um die Umsetzung in die Praxis zu erleichtern, ist die Schaffung und Implementierung eines einheitlichen MonitoringSystems speziell für die Pflege unabdingbar. Hier sind Ernährungsmedizin, Pflegewissenschaft und Medizinischer Dienst gefordert. Nur wenn alle Beteiligten eng zusammenarbeiten, kann der Ernährungszustand zum Wohle der Patienten gebessert werden.

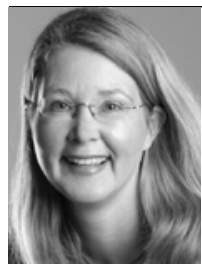

Beate Ebbers

Diplom-Oecotrophologin beate.ebbers@web.de 